Check for updates

Cite this: Nanoscale Adv., 2019, 1, 2216

\title{
Rutile nano-bio-interactions mediate dissimilar intracellular destiny in human skin cells $\dagger$
}

\author{
P. L. Sanches, (D) abc W. Souza, (D) acd S. Gemini-Piperni, (D) ${ }^{\text {ce }}$ A. L. Rossi, (D) e S. Scapin, ${ }^{a}$ \\ V. Midlej, ${ }^{f}$ Y. Sade, ${ }^{a}$ A. F. Paes Leme, ${ }^{9}$ M. Benchimol, ${ }^{\text {bf }}$ L. A. Rocha, (D) ${ }^{c h}$ R. B. V. Carias, ${ }^{i}$ \\ R. Borojevic, (iD ' J. M. Granjeiro (D) abcdj and A. R. Ribeiro (D)*bcd
}

The use of nanoparticles (NPs) in the healthcare market is growing exponentially, due to their unique physicochemical properties. Titanium dioxide nanoparticles $\left(\mathrm{TiO}_{2} \mathrm{NPs}\right)$ are used in the formulation of sunscreens, due to their photoprotective capacity, but interactions of these particles with skin cells on the nanoscale are still unexplored. In the present study we aimed to determine whether the initial nanobiological interactions, namely the formation of a nano-bio-complex (other than the protein corona), can predict rutile internalization and intracellular trafficking in primary human fibroblasts and keratinocytes. Results showed no significant effect of NPs on fibroblast and keratinocyte viability, but cell proliferation was possibly compromised due to nano-bio-interactions. The bio-complex formation is dependent upon the chemistry of the biological media and NPs' physicochemical properties, facilitating NP internalization and triggering autophagy in both cell types. For the first time, we observed that the intracellular traffic of NPs is different when comparing the two skin cell models, and we detected NPs within multivesicular bodies (MVBs) of keratinocytes. These structures grant selected input of molecules involved in the biogenesis of exosomes, responsible for cell communication and, potentially, structural equilibrium in human tissues. Nanoparticle-mediated alterations of exosome quality, quantity and function can be another major source of nanotoxicity.

Received 8th February 2019

Accepted 10th April 2019

DOI: 10.1039/c9na00078j

rsc.li/nanoscale-advances

\section{Introduction}

It is estimated that 400000 workers worldwide are working in the field of nanotechnology, being in direct contact with nanostructures during synthesis, handling, use or disposal. The Consumer Product Inventory ${ }^{1}$ refers to titanium dioxide nanoparticles $\left(\mathrm{TiO}_{2} \mathrm{NPs}\right)$ as some of the predominant nanomaterials synthesized and present in consumer products such as cosmetics, food colorants and nutritional supplements. As a consequence, exposure of humans to $\mathrm{TiO}_{2} \mathrm{NPs}$ occurs mainly by inhalation, oral or dermal exposure. ${ }^{2}$ In cosmetics, $\mathrm{TiO}_{2} \mathrm{NPs}$ are regularly used as inorganic physical sun-blockers due to their photoprotective capacity. ${ }^{3-7}$ The advantage of using nanosized $\mathrm{TiO}_{2}$ NPs is their transparency to visible light (when compared with micron-sized particles that are opaque) while providing suitable solar protection..$^{3-7}$ According to the European legislation, ${ }^{8} \mathrm{TiO}_{2}$ NPs may be used as an inorganic UV filter in sunscreen products, with a maximum content of up to $25 \%(\mathrm{wt} / \mathrm{wt})$. The literature reports that micron-sized $\mathrm{TiO}_{2}$ particles are biologically inert for both humans and animals, since they do not penetrate the skin. ${ }^{9-13}$ However, due to a higher surface area to volume ratio, NPs are more (bio)reactive compared to micrometric particles. ${ }^{2,14} \mathrm{TiO}_{2}$ NP penetration may
${ }^{a}$ Directory of Life Sciences Applied Metrology, National Institute of Metrology Quality and Technology, Av. Nossa Senhora das Graças 50, Xérem, Rio de Janeiro, Brazil

${ }^{b}$ Postgraduate Program in Translational Biomedicine, University of Grande Rio, Rua Prof. José de Souza Herdy, 1160, Duque de Caxias, Brazil. E-mail: analopes0781@ gmail.com

${ }^{c}$ Brazilian Branch of Institute of Biomaterials, Tribocorrosion and Nanomedicine (IBTN), Brazil

${ }^{d}$ Postgraduate Program in Biotechnology, National Institute of Metrology Quality and Technology, Av. Nossa Senhora das Graças 50, Xérem, Rio de Janeiro, Brazil

${ }^{e}$ Brazilian Center for Research in Physics, R. Dr. Xavier Sigaud, 150 - Urca, Rio de Janeiro, Brazil

${ }^{f}$ Instituto de Biofisica Carlos Chagas Filho, Universidade Federal do Rio de Janeiro, Av. Brg. Trompowski, Rio de Janeiro, Brazil
${ }^{g}$ Laboratório Nacional de Biociências, CNPEM, R. Giuseppe Máximo Scolfaro, 10000 - Polo II de Alta Tecnologia de Campinas - Campinas/SP, Brasil Bosque das Palmeiras, Campinas, São Paulo, Brazil

${ }^{h}$ Physics Department, University Estadual Paulista, Av. Eng. Luiz Edmundo Carrijo Coube, Bauru, São Paulo, Brazil

${ }^{i}$ Center of Regenerative Medicine, Faculty of Medicine, FASE, Av. Barão do Rio Branco 1003, Petrópolis, RJ, Brazil

${ }^{j}$ Dental School, Fluminense Federal University, R. Miguel de Frias, 9 - Icarai, Niterói, Brazil

$\dagger$ Electronic supplementary information (ESI) available: Bio-nano interaction information: tables with a detailed 'minimum information standard' for studies investigating bio-nano interactions. Stability of $\mathrm{TiO}_{2}$ NPs: a table showing $\mathrm{TiO}_{2}$ NPs' hydrodynamic size upon $24 \mathrm{~h}$ of stability testing in cell culture medium. See DOI: 10.1039/c9na00078j 
also be favoured in damaged skin (in the case of wound healing or sunburnt skin). ${ }^{6,15-17}$

It is known that the response of biological systems to NPs depends on their physicochemical characteristics in the biological environment. ${ }^{18-21}$ The size, shape, composition, ionic dissolution and crystal structure of NPs directly cause specific biological consequences. ${ }^{20,22-24}$ In vitro studies suggest that $\mathrm{TiO}_{2}$ NPs have toxic effects on many skin cell models. ${ }^{2,10,14,25,26}$ The major mechanism of toxicity is suggested to be oxidative stress, since $\mathrm{TiO}_{2}$ NPs trigger the formation of reactive oxygen species (ROS), which can induce cell death.,26,27 Tucci et al. and Shukla et al. confirmed the involvement of ROS in DNA damage of human epidermal and HaCaT cells (keratinocyte cell lineage). ${ }^{25,28,29}$ The relationship between $\mathrm{TiO}_{2}$ NPs and DNA damage in human dermal fibroblasts has also been reported. ${ }^{30}$ At a lower exposure dose, $\mathrm{TiO}_{2}$ NPs can also induce autophagy in primary human keratinocytes, protecting them against cell damage and death..$^{10}$ Nevertheless, most of the available literature assessed micron-sized $\mathrm{TiO}_{2}$ agglomerates' cytotoxicity, without testing nanostructures and not taking into consideration the nano-bio interactions.

The role of the NP interface with biological systems in nanotoxicity remains unresolved. Once in contact with biological fluids, NPs are exposed to a complex mixture of proteins able to form a protein corona. This corona is considered as the biological identity of NPs and it is what cells sense. The protein corona has become a common subject of research due to its role in determining the efficiency, efficacy, and the ultimate biological fate of nanoparticles. ${ }^{23,27-30,40}$ Also it is known to mediate cellular responses such as cellular uptake, accumulation, intracellular localization, and biodistribution. ${ }^{18,31-34}$ It is also important to stress that NP surfaces can induce conformational changes in adsorbed protein molecules which may affect the overall bio-reactivity of the NPs. ${ }^{27,41}$ However, the composition of this bio interface is even more complex, and not exclusive of protein adsorption. ${ }^{35,36}$ Recently, Ribeiro et al. reported that besides a protein corona, $\mathrm{TiO}_{2} \mathrm{NPs}$ are surrounded by an ionic shell that mediates NP internalization into human bone cells. $^{37,38}$ The current state of knowledge apparently is not sufficient to elucidate mechanisms of the nano-bio interactions of $\mathrm{TiO}_{2} \mathrm{NPs}$ and skin cells.

In the present study, we have addressed the nano-bio interactions of $\mathrm{TiO}_{2}$ rutile NPs in the primary cultures of human fibroblasts and keratinocytes. Most of the published studies use transformed cell lines, which may have different sensitivity to NPs, compared to primary cell cultures. Proteomics, electron microscopy, and specific biological assays were used to show the formation of a nano-bio-complex that enables $\mathrm{TiO}_{2} \mathrm{NP}$ internalization and intracellular trafficking, and may induce autophagy in skin cells.

\section{Experimental section}

$\mathrm{TiO}_{2}$ NP dry powder (SkySpring Nanomaterials, USA, Product no. 7920DL, particles: size $10-30 \mathrm{~nm}$, density $4.23 \mathrm{~g} \mathrm{~cm}^{-3}$, specific surface area $\sim 50 \mathrm{~m}^{2} \mathrm{~g}^{-1}$ ) was used in all experiments. We used Dulbecco's Modified Eagle's Medium high glucose
(Lonza 0378377) with 10\% foetal bovine serum (FBS, GIBCO 12657029) and KGM (Lonza, CC-3101 \& CC-4131 - KGM BulletKit) with $10 \%$ FBS. Bovine serum albumin (BSA fraction V, Sigma-Aldrich, 904846-8) was used as a stabilizing agent during the optimization protocol of rutile dispersion. A detailed 'minimum information standard' for studies investigating bionano interactions was implemented in this study in order to improve reproducibility (ESI†).

\section{Physicochemical characterization of rutile nanoparticles}

The following powder characteristics were verified before the experiments started: particle size and morphology were analyzed using a high-resolution transmission electron microscope (HRTEM, JEOL 2100F operating at $200 \mathrm{kV}$ ). The Brunauer-Emmet-Teller (BET) specific surface area of the rutile powder was determined using an Autosorb-1, Quanta Chrome Instruments. The phase composition of rutile NPs was determined using selected area electron diffraction (SAED) patterns obtained using an electron microscope (JEOL 2100F, $200 \mathrm{kV}$ ). For all TEM analyses, a drop of the $\mathrm{TiO}_{2}$ aqueous suspension was placed onto a holey carbon-coated copper grid, air-dried and observed with a TEM.

\section{Optimization of a dispersion protocol}

The strategy employed in this work for rutile dispersions in biological medium had two basic procedures: (1) powder dispersion by sonication in distilled water (stock suspension), (2) dilution of stock suspension in cell culture media (DMEM or KGM) using a stabilizing agent such as BSA. Following ISO 22412 (2008), we have chosen the cumulative method in which the mean particle size and the polydispersity index (PDI) are calculated. Mean particle size and polydispersity index (PDI) were investigated by dynamic light scattering (DLS) using a ZetaSizer Nano ZS (Malvern Instruments GmbH, Germany). DLS measurements were executed at $25{ }^{\circ} \mathrm{C}$ using standard $10 \mathrm{~mm}$ disposable optical polystyrene cuvettes. Each run of DLS measurements was automatically determined by the instrument's software. For all samples, the suspension was homogenized by pipetting up and down several times before filling the sample in the cuvette. All the suspensions were also characterized by TEM.

In distilled water. A concentration of $10 \mathrm{mg} \mathrm{mL}{ }^{-1}$ of $\mathrm{TiO}_{2}$ NPs was prepared in distilled water. NP suspensions were then dispersed using indirect mode in a cup type sonicator (Q-Sonica $700 \mathrm{~W}$, USA) at different amplitudes, times and regimes of sonication. All the dispersions were performed in a water bath with ice to prevent sample heating. Due to Eppendorf vial size variability (data not shown) a stock solution was prepared. After 24 hours of stabilization, the size distribution of particles was determined by DLS and size and morphology were evaluated by TEM.

In culture medium. After achieving the best dispersion conditions in distilled water $(30 \%$ amplitude, 15 minutes of pulsed mode), tests with culture media were performed as follows. The dispersed rutile stock solution stabilized for 24 hours was diluted to a concentration of $1 \mathrm{mg} \mathrm{mL}^{-1}$ in the 
specific culture media (DMEM High glucose 10\% FBS and KGM). As a stabilizing agent, BSA was used and the concentration was optimized for each medium. BSA at a final concentration of $0.6 \mathrm{mg} \mathrm{mL}^{-1}$ for DMEM and $72.5 \mathrm{mg} \mathrm{mL}^{-1}$ to KGM was added to the suspension before incubation in culture media. The size distribution of $\mathrm{TiO}_{2}$ NPs and the stability of the dispersion after $24 \mathrm{~h}$ were determined by DLS (ESI $\dagger$ ).

\section{Rutile nano-bio complex formation}

Rutile suspensions in culture medium supplemented with BSA were investigated by high-resolution transmission electron microscopy (HRTEM), using a TITAN 80-300 electron microscope (FEI, Netherlands) operating at $200 \mathrm{kV}$ and equipped with an energy dispersive spectroscopy (EDS) detector. The EDS maps of NPs were obtained in scanning transmission electron microscopy (STEM) mode. The samples were prepared by dropping the rutile suspensions diluted in culture media as described onto a holey coated copper grid. After naturally drying in air, the samples were analyzed.

\section{Proteomics analysis}

$100 \mu \mathrm{g} \mathrm{mL}^{-1}$ of rutile NP suspensions in DMEM with $10 \% \mathrm{FBS}$, DMEM 10\% FBS (positive control) and DMEM without FBS (negative control) were incubated for $1 \mathrm{~h}$ at $37^{\circ} \mathrm{C}$ with $5 \% \mathrm{CO}_{2}$. $100 \mu \mathrm{g} \mathrm{mL}{ }^{-1}$ rutile NPs diluted in KGM, KGM with $10 \%$ FBS (positive control) and KGM without FBS (negative control) were incubated for $1 \mathrm{~h}$ at $37^{\circ} \mathrm{C}$ with $5 \% \mathrm{CO}_{2}$. After the incubation, the samples were ultra-centrifuged at $30966 \mathrm{~g}$, for $60 \mathrm{~min}$ at $4{ }^{\circ} \mathrm{C}$, and the pellet was washed twice and suspended in $200 \mu \mathrm{L} 1 \times$ buffer (Tris-HCl $0.0625 \mathrm{M}$, SDS 2.5\%, 5\% 2-mercaptoethanol, $7 \%$ glycerol). Then the samples were frozen at $-20{ }^{\circ} \mathrm{C}$. Protein adsorption to rutile suspensions in culture medium was investigated by SDS-PAGE. The samples were boiled for $10 \mathrm{~min}$ at $100{ }^{\circ} \mathrm{C}$ and run in $7.5 \%$ bis-polyacrylamide gel, $120 \mathrm{~V}$, to separate proteins by molecular weight. The gel was stained using colloidal Coomassie blue (BIO-RAD 161-0406).

\section{Protein digest preparation and mass spectrometry identification}

The protein bands were excised from the gel, de-stained and digested in a gel using trypsin. Briefly, the proteins were reduced (10 $\mathrm{mM}$ DTT, $30 \mathrm{~min}, 25{ }^{\circ} \mathrm{C}$ ) and alkylated (iodoacetamide $50 \mathrm{mM}, 25{ }^{\circ} \mathrm{C}, 30 \mathrm{~min}$ in the dark), followed by sample digestion with $0.2 \mu \mathrm{g}$ sequencing grade modified trypsin (Promega, V5111) in $50 \mathrm{mM}$ ammonium bicarbonate, $30 \mathrm{~min}$ on ice and overnight at $37^{\circ} \mathrm{C}$. The extracted tryptic peptide mixture was then lyophilized, dissolved in $1 \%$ formic acid and subjected to StageTip C18. The samples were dried in a vacuum concentrator and reconstituted in $20-50 \mu \mathrm{L}$ of $0.1 \%$ formic acid. Aliquots of $4.5 \mu \mathrm{L}$ were analyzed on an ETD-enabled OrbitrapVelos mass spectrometer (Thermo Fisher Scientific, Waltham, MA, USA) connected to an EASY-nLC system (ProxeonBiosystem, West Palm Beach, FL, USA) through a Proxeonnanoelectrospray ion source. Peptides were separated using 2$90 \%$ acetonitrile gradient in $0.1 \%$ formic acid using an analytical column, PicoFrit column $(20 \mathrm{~cm} \times$ ID $75 \mu \mathrm{m}, 5 \mu \mathrm{m}$ particle size, New Objective), with a flow rate of $300 \mathrm{~nL} \min ^{-1}$ over $27 \mathrm{~min}$. The nanoelectrospray voltage and the source temperature were set to $2.2 \mathrm{kV}$ and $275{ }^{\circ} \mathrm{C}$, respectively. All instrument methods for the LTQ OrbitrapVelos were set up in datadependent analysis (DDA) mode. Full scan MS spectra $(\mathrm{m} / \mathrm{z}$ 300-1600) were acquired on an Orbitrap analyzer after accumulation to a target value of $1^{6}$. The resolution in the Orbitrap analyzer was set to $r=60000$. The 20 most intense peptide ions with charge states $\geq 2$ were sequentially isolated to a target value of 5000 and fragmented in a linear ion trap by low-energy CID (normalized collision energy of 35\%). The signal threshold for triggering an MS/MS event was set to 1000 counts. Dynamic exclusion was enabled with an exclusion size list of 500 and an exclusion duration of $60 \mathrm{~s}$. The activation $Q$-value was 0.25 and the activation time was $10 \mathrm{~ms}$. Data were acquired using the Xcalibur software package and samples were analyzed in three biological replicates. Peak lists (msf) were generated from the raw data files using Proteome Discoverer version 1.3 (Thermo Fisher Scientific) with the Sequest search engine and searched against bovine taxon of the UniProtKB/SwissProt database (release 2016_04) with carbamidomethylation as a fixed modification, oxidation of methionine as a variable modification, one trypsin missed cleavage, a parent ion tolerance of $10 \mathrm{ppm}$ and a fragment ion mass tolerance of 1 Da. Scaffold (version Scaffold_4.5.1, Proteome Software Inc., Portland, OR) was used to validate MS/MS based peptide and protein identifications. Peptide identifications were accepted if they could be established at greater than $99.0 \%$ probability to achieve an FDR less than $1.0 \%$ using the Scaffold Local FDR algorithm. Protein identifications were accepted if they could be established at greater than $80.0 \%$ probability to achieve an FDR less than $1.0 \%$ and contained at least 1 identified peptide. Protein probabilities were assigned using the Protein Prophet algorithm. Proteins that contained similar peptides and could not be differentiated based on MS/MS analysis alone were grouped to satisfy the principles of parsimony. Proteins sharing significant peptide evidence were grouped into clusters. Proteins were annotated with GO terms from Bostaurus filtered annotation file (gene_association.goa_cow.gz, downloaded 06-Apr-2016).

\section{Cell culture}

In vitro skin cell models were employed since in dermal applications this potentially represents the most likely route of exposure to $\mathrm{TiO}_{2}$ NPs. Primary human fibroblasts and keratinocytes were obtained from Rio de Janeiro Bio-Bank (BBRJ APABCAM, INMETRO Technological Park, Rio de Janeiro, Brazil), which obtained both cell types from preputial skin explants of patients and certified cell origin, identification, and absence of any contaminant. All the procedures were carried out after getting approval from the local ethical committee (University of Grande Rio, Ethic Committee's register numbers: 4679915.1.300.5282). After isolation and characterization, 3000 cells per $\mathrm{cm}^{2}$ of fibroblasts and keratinocytes were plated and incubated in Dulbecco's Modified Eagle's Medium high glucose, with $10 \% \mathrm{FBS}$, and $\mathrm{KGM}$, for $24 \mathrm{~h}$ at $37{ }^{\circ} \mathrm{C}$, with $5 \%$ $\mathrm{CO}_{2}$. After 2 passages, the cells, at approximately $70 \%$ 
confluency, were incubated with rutile nanoparticles at 10, 46.4 and $100 \mu \mathrm{g} \mathrm{mL} \mathrm{mL}^{-1}$ concentrations for $48 \mathrm{~h}$. The maximum number of cell passages used was 5 passages for keratinocytes and 6 passages for fibroblasts. Potential mycoplasma contamination was controlled by bioluminescence, using the MycoAlert $^{\mathrm{TM}}$ PLUS kit Mycoplasma Detection (MycoAlert ${ }^{\circledR}$, Lonza).

\section{Cell viability studies}

The cell viability assay was carried out using flow cytometry. Cells were cultured in $75 \mathrm{~cm}^{2}$ cell culture flasks (CELLSTAR®) Filter Cap Cell Culture Flasks, Greiner Bio One) for 24 hours, followed by exposure to $10,46.4$ and $100 \mu \mathrm{g} \mathrm{mL} \mathrm{mL}^{-1}$ rutile nanoparticles for 48 hours. Cell death or apoptosis was quantified on $2 \times 10^{5}$ cells using a dead cell apoptosis kit for annexin V (Life Technologies, Willow Creek Road, Eugene, OR, USA). The samples were incubated for $15 \mathrm{~min}$ in $3 \mu \mathrm{L}$ annexin/FITC solution and $1 \mu \mathrm{L}$ propidium iodide. All analyses were carried out using a FACSAria III flow cytometer (DB, Qume Drive San Jose, CA, USA).

\section{Nanoparticle internalization studies}

Primary human fibroblasts and keratinocytes were incubated with rutile nanoparticles at $10,46.4$ and $100 \mu \mathrm{g} \mathrm{mL} \mathrm{L}^{-1}$ concentrations for $48 \mathrm{~h}$ and processed for transmission electron microscopy analysis as described in Ribeiro et al. ${ }^{37}$ For lysosome identification, we used acid phosphatase detection. After the samples were fixed, they were washed 3 times with Tris-maleate buffer (0.2 M; pH 5.0) and incubated for $20 \mathrm{~min}$ at $37^{\circ} \mathrm{C}$, in a medium containing $11.5 \mathrm{mM}$ sodium $\beta$-glycerophosphate as the substrate, $2.4 \mathrm{mM}$ lead nitrate, and $5 \%$ sucrose in $0.1 \mathrm{M}$ acetate buffer (pH 5.0). This cytochemistry of acid phosphatase was performed with a modified method of Robinson and Karnovsky (1983), ${ }^{42}$ and the samples were processed for transmission electron microscopy.

\section{Statistical analysis}

All the experiments were performed at least in triplicate. A response of " $100 \%$ viability" was considered using values of the untreated controls. Means and standard deviations were calculated, and statistical significance was evaluated using the $t$ test (SigmaPlot), $P<0.05$.

\section{Results and discussion}

Physicochemical characterization of rutile nanoparticles

Commercially available rutile nanoparticles were used to mimic the NPs found in sunscreens. The physicochemical characterization of rutile NPs indicates that they were agglomerated in water (Fig. 1a). The high-resolution TEM images showed the spindle-shape of individual NPs, which present a primary size of approximately $21 \mathrm{~nm}$ (Fig. $1 \mathrm{~b}$ and c). Their specific surface area measured by BET was $26.96 \pm 0.7 \mathrm{~m}^{2} \mathrm{~g}^{-1}$ and the phase composition of rutile NPs was confirmed using selected area

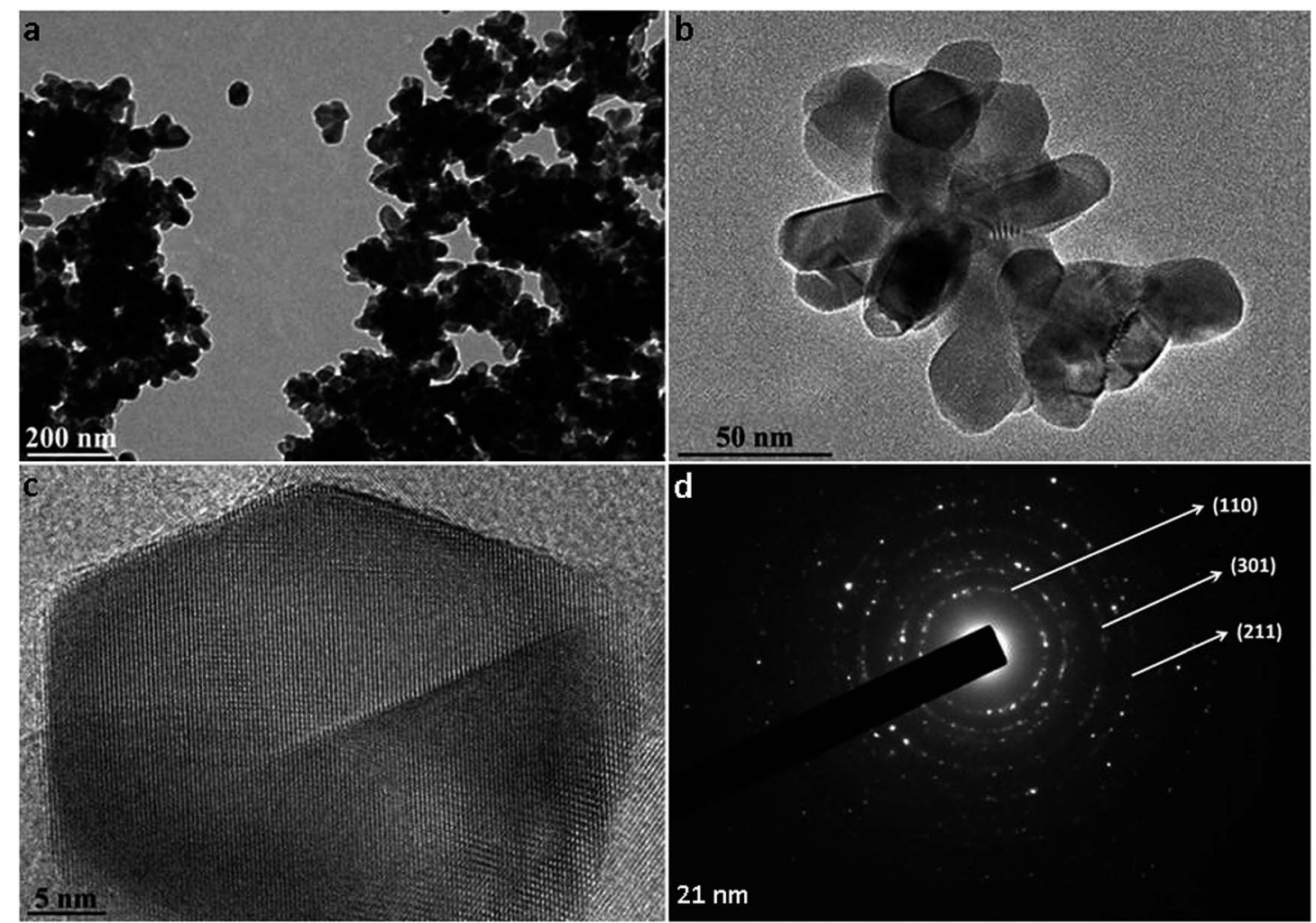

Fig. 1 TEM characterization of rutile nanoparticles: (a) image of rutile agglomerates, (b) small agglomerates and (c) HRTEM of isolated particles; (d) SAED indicating the rutile phase. 
electron diffraction (SAED) patterns (Fig. 1d). A mean hydrodynamic size of $477.7 \pm 25.2 \mathrm{~nm}$ was measured by dynamic light scattering for rutile NPs without dispersion. When nanoparticles came into contact with aqueous media, they tended to agglomerate into micrometre sized structures, with potentially different biological properties compared to nanosized structures. $^{21,39}$ Sonication is the most widely used procedure to disperse nanomaterials in toxicological studies, since the cavitation effect induced by ultrasound facilitates the disruption of agglomerates. ${ }^{\mathbf{4 0 , 4 1}}$ Taking this into consideration, we used an indirect probe sonicator (cup-horn) ${ }^{43}$ to disperse rutile nanoparticles. A suitable dispersion protocol of rutile NPs in water was developed, resulting in a mean hydrodynamic size of 142.1 $\pm 5.6 \mathrm{~nm}$ (Fig. 2a). No significant variability was observed in the multiple independent dispersions prepared for all biological assays.

\section{Dispersion and nano-bio interactions of rutile nanoparticles with biological medium}

In stage two, the suspensions prepared in water were diluted in physiological media: high glucose Dulbecco's Medium (DMEM) and Keratinocyte Growth Medium (KGM), conventionally used for culture of fibroblasts and keratinocytes, respectively. The

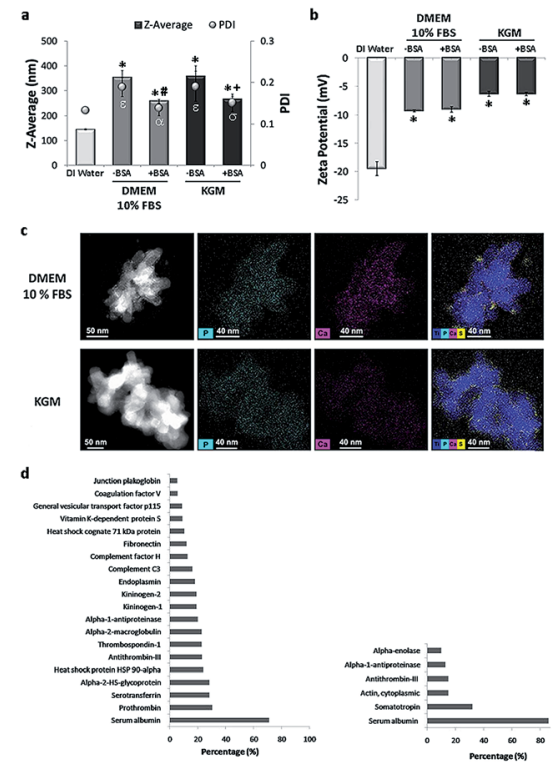

Fig. 2 Physicochemical characterization of rutile dispersions in cell culture media: (a) hydrodynamic size (diameter) and (b) potential zeta of rutile nanoparticles in DMEM 10\% FBS and KGM with or without BSA stabilization. (c) Dark-field STEM images showing where the corresponding elemental maps were obtained; STEM micrograph of rutile nanoparticles in culture medium, STEM/EDS P-K map; STEM/EDS Ca$K$ map and STEM/EDS overlay of Ti, $P, C a$, and $S$ for rutile nanoparticles in DMEM 10\% FBS and KGM. (d) Most-abundant corona proteins detectable on rutile NPs incubated with DMEM 10\% FBS and KGM. Results are mean \pm SEM of three independent experiments. Zaverage: $* P<0.05$ vs. control (DI water); $\# P<0.05$ vs. control ( $-\mathrm{BSA}$ [0 mg mL ${ }^{-1}$ ] in DMEM high glucose 10\% FBS); $+P<0.05$ vs. control (-BSA [0 mg mL ${ }^{-1}$ ] in KGM). Pdl: $\varepsilon P<0.05$ vs. control (DI water); $\alpha P<$ 0.05 vs. control (-BSA [0 $\mathrm{mg} \mathrm{mL}^{-1}$ ] in DMEM high glucose $10 \% \mathrm{FBS}$ ); $\sigma P<0.05$ vs. control (-BSA [0 $\mathrm{mg} \mathrm{mL}^{-1}$ ] in KGM). average diameter of NPs in both media increased compared to the size of the dispersion in water (Fig. 2a). The two media tested are rich in ionic species that can affect the surface charge of NPs, leading to a reduction in the electrostatic repulsive forces and causing the observed agglomeration. Both media are also rich in proteins. The different proteins from serum are expected to adsorb onto rutile NPs, contributing to agglomeration. Different dispersants have been already applied to nanoparticles, namely natural surfactants, phospholipids, organic solvents, and serum or albumin. ${ }^{35,39-41}$ Bovine serum albumin (BSA) is often used as a stabilizing agent, since it is a multifunctional protein present in plasma, and it is used in many media for toxicological studies. ${ }^{39} \mathrm{Ji}$ et al. used BSA as a protein-rich supplement to improve $\mathrm{TiO}_{2}$ NP dispersion in mammalian, bacterial, and yeast cell culture media. ${ }^{39}$ Our group recently reported that $\mathrm{TiO}_{2}$ anatase was better dispersed in BSAsupplemented medium than in conventional culture medium. ${ }^{37}$

To improve rutile NP dispersion in culture media, BSA was used as a model protein. The BSA concentration was adjusted to obtain the best rutile NP dispersion. DLS analysis showed that, at a constant rutile concentration, BSA was effective in reducing agglomeration in both cell culture media (Fig. 2a). The adsorption of BSA on rutile NP surfaces may possibly occur through electrostatic, hydrophobic, or specific chemical interactions. This is in agreement with the literature, where BSA is largely used as a stabilizing agent preventing NP agglomeration. ${ }^{42}$ No significant differences were observed in the average size of stabilized NPs in both cell culture media (DMEM: 258.2 \pm 0.13 and KGM: $266.2 \pm 0.15)$. A slight increase in the zetapotential was observed upon addition of rutile $\mathrm{TiO}_{2}$ NPs to both media (with and without BSA) (Fig. 2b). This alteration in zeta potential is probably due to the formation of a bio-complex (binding of ions and proteins from the medium culture) on the $\mathrm{TiO}_{2}$ NP surface. The ionic species adsorbed on rutile were analyzed by EDS at high resolution. STEM elemental maps (Fig. 2c) reveal the preferential adsorption of calcium (Ca), phosphorus (P), and sulfur (S) derived from the culture media on the $\mathrm{TiO}_{2}$ NP surface.

The Ca contents were lower for KGM compared to DMEM medium (0.1 $\mathrm{mM} \mathrm{Ca}^{2+}$ for KGM and $0.2 \mathrm{mM}$ for DMEM $10 \%$ FBS) in accordance with the published data. ${ }^{\mathbf{4 4 , 4 5}}$ This ionic adsorption was also observed when rutile NPs were incubated in both media without bovine serum and albumin stabilization. Although the mechanism of adsorption appears to be independent of the presence of proteins, they are possibly contributing to the enhancement of ion binding. Ionic adsorption appears to be dependent on the rutile surface properties. The presence of hydroxyl groups on the rutile surface possibly provides sites for calcium and phosphate adsorption through electrostatic forces. After $\mathrm{Ca}^{2+}$ adsorption to $\mathrm{OH}^{-}$groups, the negatively charged phosphate ions $\mathrm{PO}_{4}{ }^{3-}$ combine with the positively charged surface. No significant differences were found in S content which confirms the adsorption of the protein corona. Possibly the divalent $\mathrm{Ca}^{2+}$ cations have an important role also in protein corona formation, since they can act as an effective bridge to bind negatively charged BSA molecules. ${ }^{44}$ Protein-nanoparticle interactions are influenced by factors 
such as the physicochemical properties of NPs, and by the biological micro-environments. ${ }^{18}$ The adsorption of proteins to the rutile NP surface was investigated in this study for both media (see Fig. 2d). SDS gel electrophoresis (data not shown) demonstrated the common and different proteins adsorbed on rutile $\mathrm{TiO}_{2}$ NPs in both media. Mass spectrometry revealed a total of 57 proteins in DMEM and 6 in KGM, respectively. The protein corona of $\mathrm{TiO}_{2} \mathrm{NPs}$ harbors albumin as one of the major constituents, used as a stabilizing agent. Albumin is involved in the transport of drugs and free radicals, in scavenging and in maintaining osmotic pressure. The preferred association of albumin with titanium dioxide surfaces and its capacity to bind with various substrates are well known and have been reported previously. ${ }^{43}$ Besides albumin, the NP corona contained the expected plasma proteins, belonging to the coagulation cascade, such as prothrombin and alpha-2-macroglobulin, or to the complement group. The presence of two other major proteins may be relevant. Thrombospondin is a glycoprotein mediating cell-cell and cell-matrix interactions being involved in platelet aggregation, angiogenesis and tumorigenesis. Fibronectin is either produced in the liver and released to blood circulation or is produced in situ by tissue-dwelling cells such as fibroblasts, immobilized on the extracellular matrix, and/or released into the circulation which often occurs under cell stress conditions. Both thrombospondin and fibronectin are calcium-binding proteins, possibly contributing to the calcium shell formation around $\mathrm{TiO}_{2}$ NPs. They establish by this divalent interaction the first adhesive contact of the cell membrane with inorganic or organic substrates. They are also ligands for integrins, which mediate cell adhesion to the extracellular matrix and mediate particle internalization into the cells by the ligand-dependent phagocytosis. ${ }^{31-34}$

The number of peptides identified in KGM was lower and less diverse compared to that in DMEM. Besides albumin, the main identified protein was somatotropin, the hormone that stimulates cell growth and reproduction, involved in cell regeneration in humans. Cytoplasmic actin-1 was also detected, and this protein is involved in cell motility, and in cell membrane movements associated with macro-phagocytosis. Peptides of the coagulation cascade were also detected in KGM, namely antithrombin-III, a naturally occurring anticoagulant protein that binds to heparin blocking thrombin and therefore inhibits fibrin clot formation. Alpha-enolase is a multifunctional enzyme that, besides its role in glycolysis, is involved in various processes namely cell growth, hypoxia, and allergic responses, stimulating immunoglobulin production. The functions associated with the NP protein corona include calcium ion binding, cell cycle control, systemic inflammation and immune response. ${ }^{31-34}$ In this context, the major proteins of the corona that are part of the bio-complex formed on its surface may be relevant for NP internalization, their fate within the cell, and their effect on cell biology and/or pathology.

\section{Biological consequences of NP exposure}

We used primary in vitro cultures of human keratinocytes and fibroblasts, the major cell types present in the human skin, as a model of the cellular response to $\mathrm{TiO}_{2}$ NPs. Our results showed alteration in both cells' morphology and a reduction in cell proliferation, in a dose dependent manner (Fig. 3a and b). The effect was more prominent on fibroblasts. At the tested concentrations, the cell viability (Fig. $3 \mathrm{c}$ and d) and cell cycle (Fig. 3e and f) were not compromised by NP exposure. The most general signalling cascades essential for cell proliferative responses are those intermediated by extracellular and intracellular calcium, $\mathrm{Ca}^{2+}$, levels. ${ }^{46}$ The media containing low calcium decrease human fibroblast cellular division, causing the accumulation of cells in the G1 phase of the cell cycle. Comparable behaviour was already reported regarding NPs' effect on keratinocyte cell culture. ${ }^{47}$ The proposed explanation was $\mathrm{Ca}^{2+}$ deregulation due to NP internalization with a consequent decrease in cell proliferation associated with an early keratinocyte differentiation, without visible cell death. Variations in $\mathrm{Ca}^{2+}$ have been already described to be related to cytotoxic effects in diverse cell models induced by the uptake of different NPs including titanium dioxide..$^{48}$ The $\mathrm{Ca}, \mathrm{P}$ and $\mathrm{S}$ rich bio-complexes, described previously, can contribute to the reduction of cell proliferation, and the differences observed here between the two cell types can be related to the quantity of $\mathrm{Ca}^{2+}$, which is different in the two studied cell media. The fact that we observed higher $\mathrm{Ca}^{2+}$ levels in the bio-complex formed in the fibroblast cell culture medium may explain their cellular effect at lower concentrations of NP exposure. Our group has already demonstrated that the $\mathrm{Ca}^{2+}$ adsorbed on $\mathrm{TiO}_{2} \mathrm{NPs}$ can be dissociated inside the cell, and this can lead to alterations in cell behaviour. ${ }^{37}$ Until now, it is unclear how the $\mathrm{Ca}$ and $\mathrm{P}$ detaching processes occur, but we could understand that they decreased skin cell proliferation without compromising cell viability.

$\mathrm{TiO}_{2}$ NPs have not been reported to cause irritation or degeneration of skin cells, and data on humans demonstrate that they have no sensitizing effect on the skin.,.$^{2,10,47,48}$ We have described above that the bio-complexes formed in the corona around $\mathrm{TiO}_{2}$ NPs may interact with cell membranes, and possibly modify or control the NP internalization into cells. Therefore, we have done an ultrastructural study of NP internalization using primary human fibroblasts and keratinocytes. Transmission electron microscopy confirmed the potential role of the NP bio-complex in cell behaviour, showing their internalization in both human skin cell types (Fig. 4).

Nanoparticle internalization in skin cell lineages was already observed and documented in the literature. ${ }^{10,48,49}$ The majority of NPs are internalized into membrane-bound vesicles (endosomes and phagosomes). Following their maturation, their contents are organised and packed for specific destinations, where exocytosis or transcytosis from the cells can occur. In our case, the two major human skin cell types, whose function and tissue distribution in the skin are quite different, have also shown differences in NP internalization. Keratinocytes grow in vivo as avascular monolayers on a well-defined basement membrane of the dermal-epidermal junction. Their external surface is fully keratinized, and the metabolic activity of cells within the underlying keratinizing layers is low, being mostly nourished by molecular diffusion from the underlying tissue. 
a

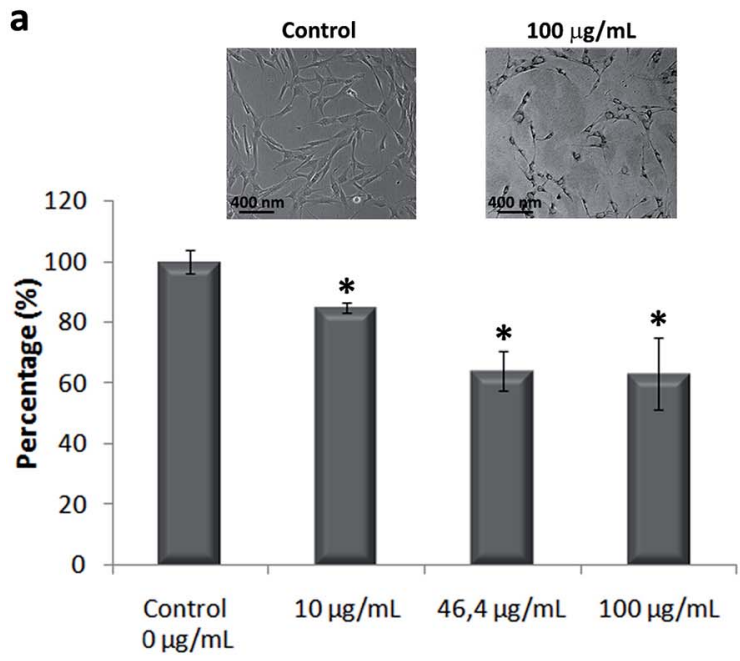

C

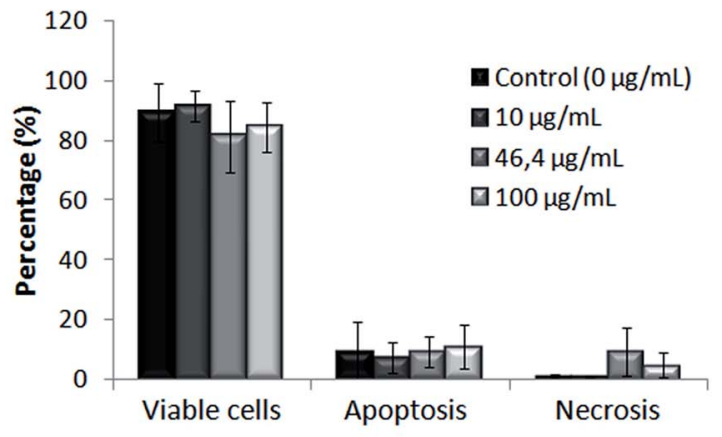

e

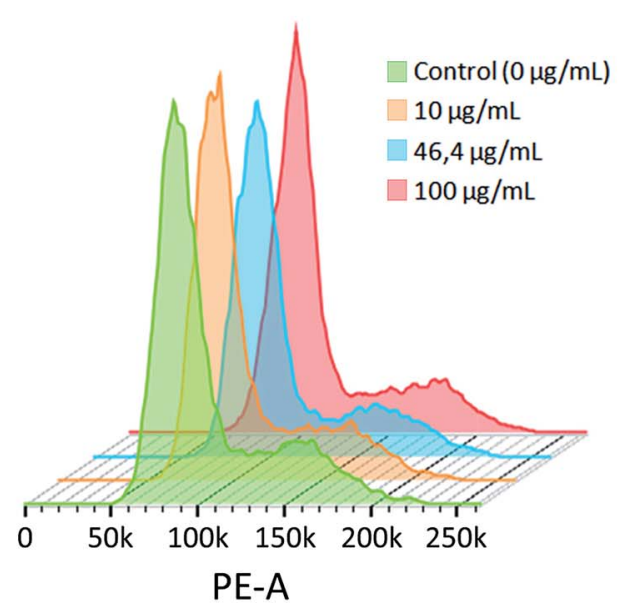

b

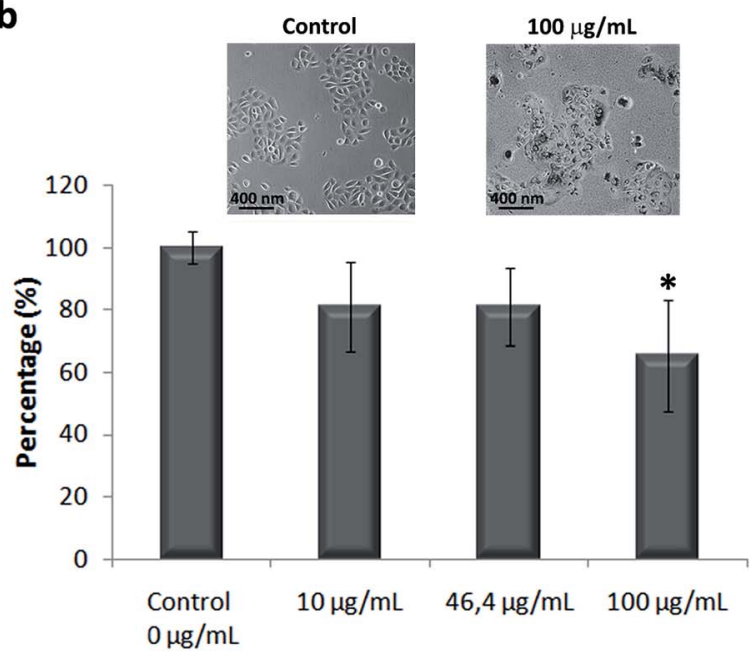

d

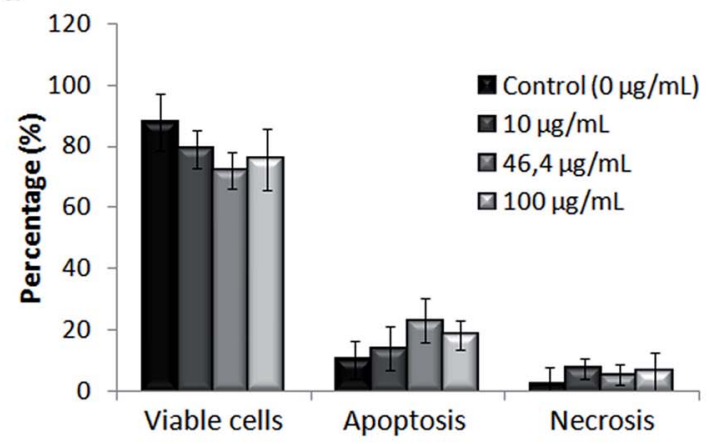

$\mathbf{f}$

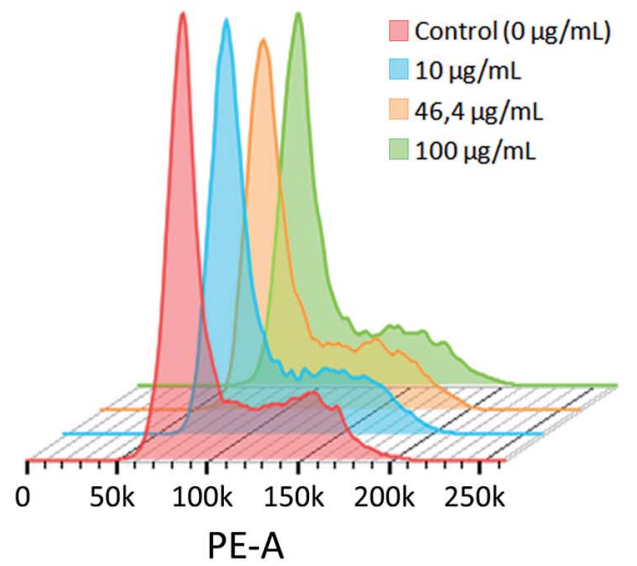

Fig. 3 Cell morphology, proliferation and viability upon exposure to $\mathrm{TiO}_{2} \mathrm{NPs}$ : (a) fibroblast and (b) keratinocyte proliferation with phase contrast images demonstrating alterations in the cell morphology. Graphs showing the percentage of viable, apoptotic and necrotic cells of fibroblasts (c) and keratinocytes (d) obtained using flow cytograms of annexin V/PI staining. (e and f) Cell cycle progression upon exposure of fibroblasts and keratinocytes to nanoparticles, respectively.

Their phagocytic activity is very low, and their major activity is the synthesis of keratin (Fig. 4a, c and e). In vitro, keratinocytes grow in monolayers and have direct contact with the culture medium. The total uptake of NPs in keratinocytes can be cumulative and quite high, but only a few images showing the first steps of internalization were obtained, only showing the uptake of single NPs, through direct and simple membrane invagination (Fig. 4a arrow). For this, submembrane actin reorganization and invagination are required, associated with the presence of other molecules underlying the membrane and required for internalization. Those involved in integrin and actin-1 activation have been identified in the corona, as 

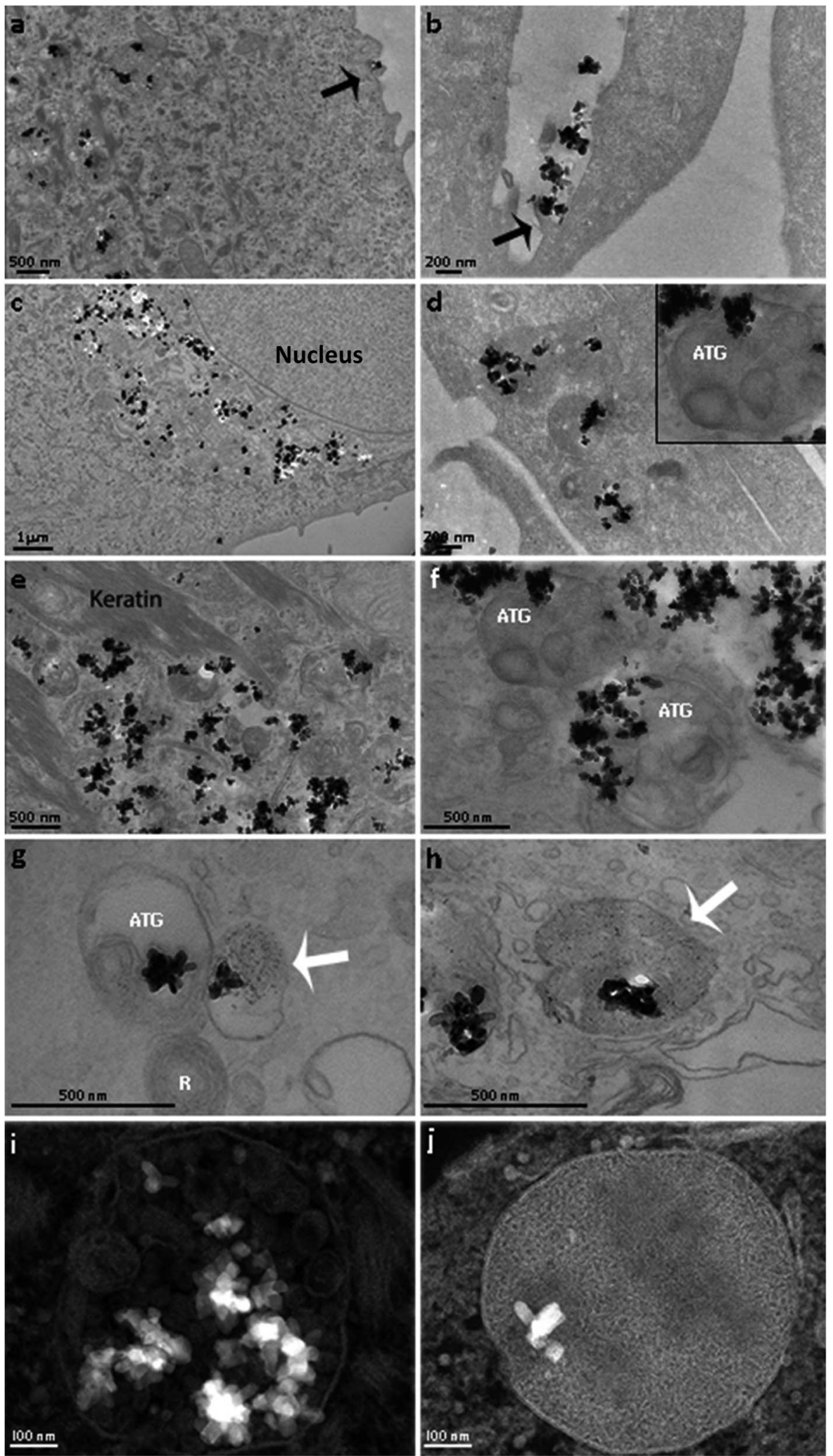

Fig. 4 TEM micrographs of primary human keratinocyte and fibroblast cells after rutile exposure: (a, c, e, $g$ and i) NPs internalized in keratinocytes and $(b, d, f, h$ and $j)$ in fibroblasts. The black arrows indicate active endocytosis with the formation of lamellipodia near NPs and the white arrows indicate the labeling of acid phosphatase. Note in figure (g) the presence of autophagolysosome (ATG) and residual body (R) in keratinocytes. Also note that nanoparticles appear to be in different vesicles in keratinocytes (I) and fibroblasts (J). 
discussed above. Primary phagosomes could also be identified within the peripheral cytoplasm, with apparently random distribution. They may be passively segregated in the perinuclear area (Fig. 4c) or they may actively follow the actin fibre bundles (Fig. 4e). $\mathrm{TiO}_{2}$ NPs were observed to be free in the cytoplasm (Fig. 4b), but they were mostly observed inside the distinct vesicles (Fig. 4e, g and i). A few vesicles were demonstrated to be lysosomes using acid phosphatase, a lysosomal enzyme marker (Fig. 4g). We could also see autophagic vacuoles demonstrating that $\mathrm{TiO}_{2}$ NP interaction can induce autophagy in primary human keratinocytes (Fig. 4g). The presence of autophagolysosomes and their association with cell death and keratinocyte autophagy were already reported in the literature. ${ }^{\mathbf{1 0 5 0}}$ They occur when the processes involve phagosomes and structures of the autophagic machinery, where cells allow the well-ordered degradation and recycling of their components. Keratinocytes potentially trigger autophagy-mediated lysosomal degradation of $\mathrm{TiO}_{2}$ NPs in order to manage NP exposure.

Surprisingly, we have also discovered isolated $\mathrm{TiO}_{2}$ NPs within multivesicular bodies (MVBs), and this potentially indicates the fusion of vesicles carrying $\mathrm{TiO}_{2}$ NPs with MVBs. Their presence in MVBs is quite important since these organelles produce exosomes. The MVB transmembrane traffic is selective, being in charge of insertion of cell-produced molecules into the exosome membrane and into the internal content. The former one is in charge of the membrane interaction with the intracellular transport mechanisms, thus being in charge of their release from the cells, followed by circulation in biological fluids, and their recognition and uptake by the surrounding or distant target-cells. The latter one is in charge of defining the content of molecules that are going to be segregated within the exosomes, destined to be delivered to the target cells. Their biological functions can be very relevant, and these nanovesicles are now known to be some of the major mechanisms for intercellular communication, allowing cells to exchange proteins, lipids and genetic material. The MVBs can fuse with lysosomes for degradation, but they also fuse with the external membrane and release their content including exosomes into the intercellular circulation. These pathways are under rigid molecular control of specific mechanisms that allow MVB secretion and consequently exosome release. Once in the extracellular space, exosomes can reach recipient cells and deliver their cargo that will affect target cell behaviour. ${ }^{51}$ We propose that the presence of $\mathrm{TiO}_{2} \mathrm{NPs}$ within the MVBs can potentially alter both the exosome membrane and the internal molecular contents. This can extensively modify in quantitative and qualitative terms the final destiny and the content of intercellular communications and cause yet unknown consequences on surrounding tissues and even on distant systemic functions. Similar behaviour was recently reported, involving amine-functionalized NPs internalized by macrophages and placed into MVBs, forming a novel membrane-bound compartment candidate for exocytosis. ${ }^{52}$

Keratinocytes are the most abundant cell type in the skin epidermis, serving as the principal barrier between the body's interior and the external environment. The MVBs with their exosomes can fundamentally contribute to the major functions of the skin, such as sensing the environment and informing the internal tissues about the external physio-chemical properties, including potential aggression and trauma, and initiating responses such as inflammation or regeneration. The fact that we observed NPs within MVBs and in direct contact with exosomes allowed us to think that exosome biogenesis, secretion, quality and quantity of the cargo can be altered, possibly contributing and leading to systemic nanotoxicity. The fusion mechanism of $\mathrm{TiO}_{2}$ NPs with MVBs, their intracellular transport, their final destiny and their potential interaction with the cell metabolism are the objectives of ongoing studies.

In contrast to keratinocytes, fibroblasts are interstitial cells of the dermis, interactive with blood vessels and with the interstitial circulation of biological fluids, involved in extracellular matrix production and turnover. They sustain complex and demanding activities of resident and circulating cells belonging to immune and phagocytic lineages. The in vitro model of fibroblast culture is less organized than that of keratinocyte monolayers, and the differences are notable (Fig. 4b, d, $\mathrm{f}, \mathrm{h}$ and $\mathrm{i}$ ). In all the observed cases, fibroblasts internalized large agglomerates of $\mathrm{TiO}_{2}$ NPs, by a phenomenon suggesting a macro-phagocytic internalization system that is not specific for chemically identified and bound ligands (Fig. 4b). This does not involve molecular recognition and apparently does not lead to the formation of autophagosomes. Some $\mathrm{TiO}_{2} \mathrm{NPs}$ were observed within autophagic vacuoles (Fig. 4f), suggesting once again that NP-containing cells activate their autophagic machinery in order to resist this NP exposure, and a few $\mathrm{TiO}_{2}$ NPs were observed inside the lysosomes (Fig. 4h). Most of the particles were located in specific membrane vesicles, completely different from keratinocytes, with a high electron density (Fig. 4j). New studies are underway in order to clarify these structures.

Comparative analysis of the $\mathrm{TiO}_{2} \mathrm{NP}$ internalization in the in vitro models of two major human skin cell populations points to two relevant phenomena. The $\mathrm{TiO}_{2}$ surface interacts specifically with inorganic and organic components of the biological environments, whose association is cumulative and interactive. Their composition and their physicochemical properties are determinant for the formation of a bio-complex, which covers the NPs and controls the nano-biological interactions with cells and surrounding tissues. Simultaneously, the cellular response to NPs and to their corona was shown to be cell type-specific. In the present study, the well-defined corona formed on $\mathrm{TiO}_{2} \mathrm{NPs}$ elicited different internalization pathways in the two major cell categories of the human skin. This points to the fact that qualitative and quantitative tolerance limits for the use of nanoparticle-containing goods, or for the environmental presence of nanoparticle compounds, have to be determined in the appropriate context of cell or tissue potentially exposed to them. When $\mathrm{TiO}_{2}$ NPs touch the exterior membrane of skin cells, they interact with the components of the plasma membrane and enter the cells mainly through endocytosis. TEM images showed active endocytosis with the formation of lamellipodia near NPs, suggesting a receptor-mediated induction of macrophagocytosis (Fig. 4a and b). Phagocytosis of $\mathrm{TiO}_{2}$ NPs in fibroblasts and keratinocytes is normally initiated by opsonins, 
such as complement proteins and fibronectin which were detected on the $\mathrm{TiO}_{2}$ NP surface through proteomics.

We consider that it is important to carefully characterize nanomaterials' physicochemical properties in biological systems before any toxicological studies in vitro. The current toxicology literature lacks data that allow toxicologists and regulators to develop accurate guidelines that could be used to assess potential hazards. There is an urgent need to standardize protocols to understand in depth the physicochemical properties of NPs, the nano-bio interface (e.g., issues affecting biomolecular corona formation), and the biological system (e.g., cell, tissue, and organ type).$^{53}$ The lack of characterization could also lead to discordant results for apparently the same tested materials, because of subtle particle differences or variances in the dispersion medium used, resulting in conflicting particle properties and toxicity results. ${ }^{24}$

Acquiring key data at the nano-bio interface is crucial in understanding the relationship between the physicochemical properties of nanomaterials and their toxicity. ${ }^{53-55}$ For a long time, scientists have been interested in studying the toxicity of a
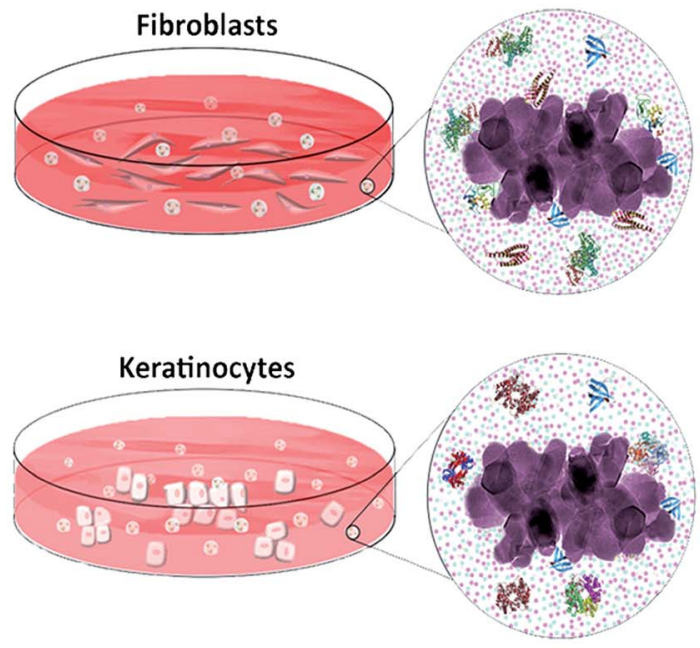

C

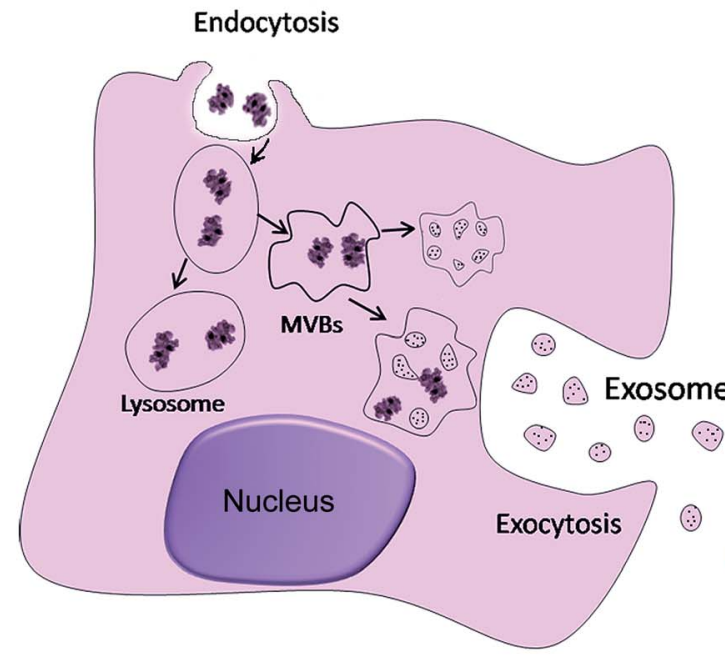

b
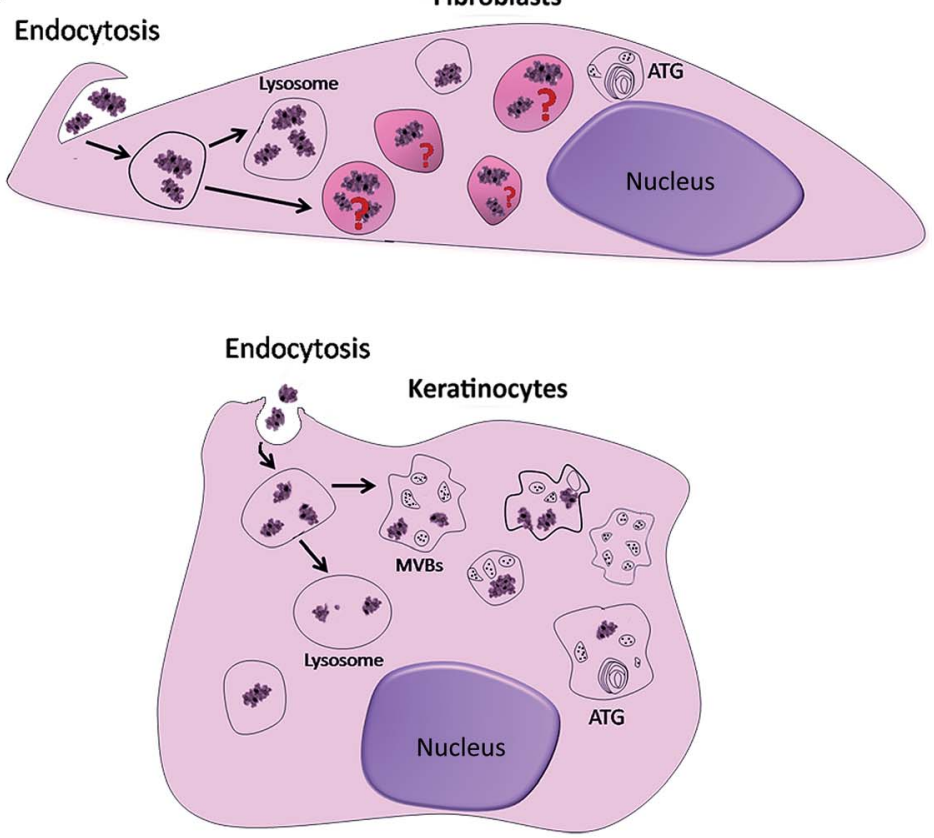

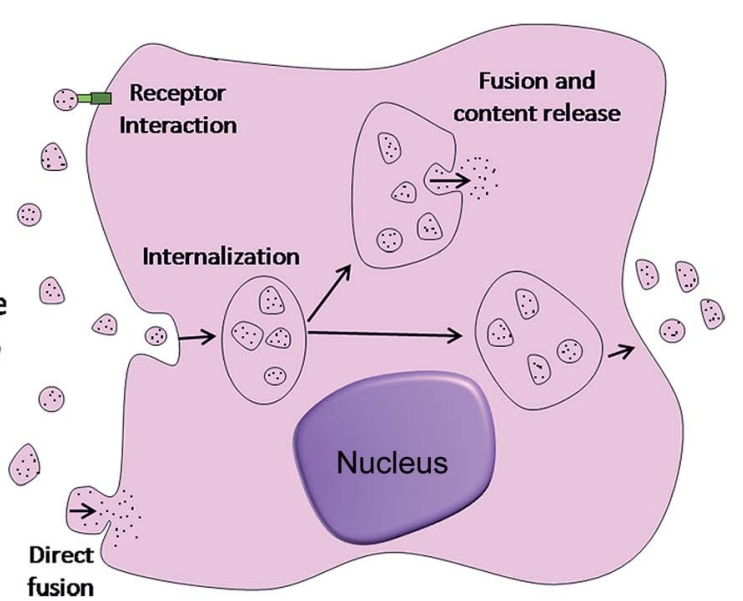

Fig. 5 Schematic model of the rutile NP bio-interface and internalization in keratinocytes and fibroblasts. (a) When rutile NPs interact with different biological media, they are instantaneously coated by proteins and by selective ions due to NP surface reactivity that will influence NP internalization and intracellular trafficking. (b) After the internalization of NPs, it can be observed that they follow different routes in keratocytes and fibroblasts. In fibroblasts, NPs were observed in lysosomes and in more electron-dense vesicles that were not yet identified (indicated with question marks), whereas in keratinocytes they were observed in lysosomes and MVBs. (c) Formation of MVBs after NP internalization, and exosome uptake by a recipient cell. 
nanomaterials in human environments without considering the nano-bio interfaces.

In the present study, we suggest that what skin cells sense and come into contact with is a bio-complex that has different characteristics according to the culture medium composition. Different contents of calcium, phosphorus and organic molecules (proteins) in the biological medium mask $\mathrm{TiO}_{2}$ nanoparticles and possibly influence their internalization, intracellular traffic and final destiny (see Fig. 5).

Up to now, the OECD report on the physicochemical properties of manufactured nanomaterials and test guidelines does not take into account the understanding of the nano-bio interface. From our point of view, the bio-complex formed around $\mathrm{TiO}_{2} \mathrm{NPs}$, through a dynamic process with different temporal patterns, can be suggested as a possible fingerprint for NPs that are recognized by skin cells (Fig. 5a). After internalizing them, they follow different routes (Fig. 5b). In the fibroblasts, as already mentioned above, NPs were encapsulated in specific vesicles such as lysosomes; however, as yet unidentified vesicles were also observed. In keratinocytes, NPs were observed in lysosomes, as expected, and in MVBs, as indicated by the arrows. In the two models studied, vesicles were observed in autophagy, autophagolysosomes (ATG). As observed in Fig. 5c, the internalization of NPs generates MVBs that fuse with the cell membrane to release exosomes in the extracellular space. According to current studies, several mechanisms have been suggested to mediate the uptake of exosomes by receptor cells, such as membrane fusion, endocytosis/phagocytosis and receptor interaction. Extracellular vesicles, including exosomes, have been already a focus of studies, since they potentially serve as biomarkers for skin diseases and can function also as specific cargo transfer vesicles, with a potential to treat dermatologic diseases. ${ }^{56}$ We believe that they can also be used to detect anomalies due to exposure of skin cells to NPs. The combined action of proteins and ions is fundamental and required for nanoparticle uptake and intracellular trafficking within the cell. This formed bio-complex may change cellular biological activities that can include the starvation state of cells and can facilitate internalization of NPs in vitro. ${ }^{33,34}$ For example, $\mathrm{Ca}$ and $\mathrm{P}$ are essential elements for cell maintenance, and their depletion due to the sequestration on the NP surface can lead to cell stress. The skin is the primary barrier against contact penetration of cosmetic products and it was important to understand the effects that are specific to these cells caused by different particles that are commonly encountered in cosmetic formulations. To translate this to consumers and to possibly predict the biological identity and readouts of these NPs, we still need to explore the biomolecular corona (protein identification, conformation and spatial location) using relevant physiological fluids and work with dynamic conditions in a three-dimensional skin model, goals that will be considered in the future.

\section{Conclusion}

In conclusion, the corona present on titanium dioxide nanoparticles is an important factor for in vitro examination of NP internalization and subsequent trafficking. In the present study, we described an anionic and protein corona when NPs were incubated with different cell culture media. Rutile nanoparticles did not interfere with the cell viability and cell cycle of primary human keratinocytes or fibroblasts, but their proliferation was compromised, possibly by the Ca content in the bio-complex formed around NPs. The interactions and association of ions and proteins with the surface of the NPs induced a similar nanoparticle internalization in primary human fibroblasts and keratinocytes. However, the intracellular trafficking was quite different, and we could conclude that keratinocytes and fibroblasts upregulate their autophagic machinery in response to NP exposure. For the first time, we report the direct contact of NPs within keratinocyte MBVs. These structures actively grant selected input of molecules involved in the biogenesis of exosomes which are the nanovesicles responsible for cellular crosstalk and for skin homeostasis. Considering the fact that there is direct contact of NPs with exosomes and that NPs release metallic ions, we can possibly think that the exosome cargo can be modified and contribute to systemic toxicity. This study highlights the necessity of carrying out physicochemical characterization of NPs in suitable physiological environments to better interpret and understand the results obtained in in vitro models. Ultimately, through the understanding of the formation and composition of these bio-complexes and their intracellular trafficking we can mitigate toxicity and selectively modulate intracellular functions and the mechanism of cell-cell transport of therapeutics for skin dermatology.

\section{Author's contribution}

A. R. R., S. G. P., L. A. R., R. B., A. F. P. L., M. B., and J. M. G. conceived and designed the experiments. P. L. S., W. S., S. G. P., S. S., R. B. V. C., V. M., P. J. B, Y. S., and A. L. R. performed the experiments. P. L. S., A. R. R., S. G. P., J. M. G., L. A. R., A. F. P. L, and R. B. analysed the data. A. R. R., J. M. G., A. F. P. L, M. B., and R. B. contributed reagents/materials/analysis tools. A. R. R. and P. L. S. wrote the paper. All authors reviewed the manuscript.

\section{Conflicts of interest}

There are no conflicts to declare.

\section{Acknowledgements}

The authors are grateful to Eveline Robertis for her valuable help in performing the BET analysis and Juliana Moscoso and Helena Cruz for their help in the optimization of the dispersion protocol. The authors acknowledge the financial support from INMETRO and from FINEP, FAPERJ (process number 111.313/ 2014APQ1 2014/01, E-26/111.313/2014), CNPq (process number 490761/2013-5) and CAPES (process number 99999.008666/2014-08). Priscila Laviola acknowledges a FAPERJ (No. 2009045272) scholarship. A. R. Ribeiro acknowledges the Jovem Cientista do Nosso Estado - 2017 and PROPESQUnigranrio/Funadesp fellowships. The authors would like to acknowledge J. Werckmann for microscopy analysis and LABNANO/CBPF for transmission electron microscopy analysis. 


\section{References}

1 M. E. Vance, et al., Nanotechnology in the real world: Redeveloping the nanomaterial consumer products inventory, Beilstein J. Nanotechnol., 2015, 6, 1769-1780.

2 H. Shi, R. Magaye, V. Castranova and J. Zhao, Titanium dioxide nanoparticles: a review of current toxicological data, Part. Fibre Toxicol., 2013, 10, 15.

3 T. Smijs and S. Pavel, Titanium dioxide and zinc oxide nanoparticles in sunscreens: focus on their safety and effectiveness, Nanotechnol., Sci. Appl., 2011, 4, 95-112.

4 M. D. Newman, M. Stotland and J. I. Ellis, The safety of nanosized particles in titanium dioxide- and zinc oxidebased sunscreens, J. Am. Acad. Dermatol., 2009, 61, 685-692.

5 Ctsuser, Nanomaterial Case Studies: Nanoscale Titanium Dioxide in Water Treatment and in Topical Sunscreen, 2010, pp. 1-204.

6 N. A. Monteiro-Riviere, et al., Safety Evaluation of Sunscreen Formulations Containing Titanium Dioxide and Zinc Oxide Nanoparticles in UVB Sunburned Skin: An In Vitro and In Vivo Study, Toxicol. Sci., 2011, 123, 264-280.

7 A. Martirosyan and Y.-J. Schneider, Engineered Nanomaterials in Food: Implications for Food Safety and Consumer Health, Int. J. Environ. Res. Public Health, 2014, 11, 5720-5750.

8 A. Salvador and A. Chisvert, Sunscreen analysis: A critical survey on UV filters determination, Anal. Chim. Acta, 2005, 537, 1-14.

$9 \mathrm{~J}$. Lademann, et al., Penetration of Titanium Dioxide Microparticles in a Sunscreen Formulation into the Horny Layer and the Follicular Orifice, Skin Pharmacol. Physiol., 1999, 12, 247-256.

10 Y. Zhao, et al., Exposure to Titanium Dioxide Nanoparticles Induces Autophagy in Primary Human Keratinocytes, Small, 2012, 9, 387-392.

$11 \mathrm{~J}$. Schulz, et al., Distribution of sunscreens on skin, Adv. Drug Delivery Rev., 2002, 54, S157-S163.

12 Y. Huang, et al., Characterization of physicochemical properties of ivy nanoparticles for cosmetic application, $J$. Nanobiotechnol., 2013, 11, 1.

13 U.S. EPA, Nanomaterial Case Studies: Nanoscale Titanium Dioxide in Water Treatment and in Topical Sunscreen (Final), U.S. Environmental Protection Agency, Washington, DC, EPA/600/R-09/057F, 2010.

14 C. Jin, et al., Cellular Toxicity of $\mathrm{TiO}_{2}$ Nanoparticles in Anatase and Rutile Crystal Phase, Biol. Trace Elem. Res., 2010, 141, 3-15.

$15 \mathrm{~J} . \mathrm{Wu}$, et al., Toxicity and penetration of $\mathrm{TiO}_{2}$ nanoparticles in hairless mice and porcine skin after subchronic dermal exposure, Toxicol. Lett., 2009, 191, 1-8.

16 A. P. Popov, J. Lademann, A. V. Priezzhev and R. Myllylä, Effect of size of $\mathrm{TiO}_{2}$ nanoparticles embedded into stratum corneum on ultraviolet-A and ultraviolet-B sun-blocking properties of the skin, J. Biomed. Opt., 2005, 10, 064037.

17 C. Bennat and C. C. Müller-Goymann, Skin penetration and stabilization of formulations containing microfine titanium dioxide as physical UV filter, Int. J. Cosmet. Sci., 2001, 22, 271-283.
18 P. Foroozandeh and A. A. Aziz, Merging Worlds of Nanomaterials and Biological Environment: Factors Governing Protein Corona Formation on Nanoparticles and Its Biological Consequences, Nanoscale Res. Lett., 2015, 10, 221.

19 S.-J. Choi and J.-H. Choy, Biokinetics of zinc oxide nanoparticles: toxicokinetics, biological fates, and protein interaction, Int. J. Nanomed., 2014, 9, 261-269.

20 G. Oberdörster, et al., Principles for characterizing the potential human health effects from exposure to nanomaterials: elements of a screening strategy, Part. Fibre Toxicol., 2005, 2, 8.

21 G. OberdÃ rster, Safety assessment for nanotechnology and nanomedicine: concepts of nanotoxicology, J. Intern. Med., 2010, 267, 89-105.

22 D. S. Cathe, J. N. Whitaker, E. K. Breitner and K. K. Comfort, Exposure to metal oxide nanoparticles in physiological fluid induced synergistic biological effects in a keratinocyte model, Toxicol. Lett., 2017, 1-7.

23 G. Oberdörster, E. Oberdörster and J. Oberdörster, Nanotoxicology: an emerging discipline evolving from studies of ultrafine particles, Environ. Health, 2005, 113, 823-839.

24 A. R. Ribeiro, et al., Challenges on the toxicological predictions of engineered nanoparticles, NanoImpact, 2017, 8, 59-72.

25 P. Tucci, et al., Metabolic effects of $\mathrm{TiO}_{2}$ nanoparticles, a common component of sunscreens and cosmetics, on human keratinocytes, Cell Death Dis., 2013, 4, e549.

26 Y. Wang, et al., Cytotoxicity, DNA damage, and apoptosis induced by titanium dioxide nanoparticles in human nonsmall cell lung cancer A549 cells, Environ. Sci. Pollut. Res., 2014, 22, 5519-5530.

27 B. Trouiller, R. Reliene, A. Westbrook, P. Solaimani and R. H. Schiestl, Titanium Dioxide Nanoparticles Induce DNA Damage and Genetic Instability In vivo in Mice, Cancer Res., 2009, 69, 8784-8789.

28 R. K. Shukla, A. Kumar, N. Vallabani and A. K. Pandey, Titanium dioxide nanoparticle-induced oxidative stress triggers DNA damage and hepatic injury in mice, Nanomedicine, 2014, 9, 1423-1434.

29 R. K. Shukla, A. Kumar, A. K. Pandey, S. S. Singh and A. Dhawan, Titanium Dioxide Nanoparticles Induce Oxidative Stress-Mediated Apoptosis in Human Keratinocyte Cells, J. Biomed. Nanotechnol., 2011, 7, 100-101. 30 Q. Saquib, et al., Titanium dioxide nanoparticles induced cytotoxicity, oxidative stress and DNA damage in human amnion epithelial (WISH) cells, Toxicol. in Vitro, 2012, 26, 351-361.

31 S. R. Saptarshi, A. Duschl and A. L. Lopata, Interaction of nanoparticles with proteins: relation to bio-reactivity of the nanoparticle, J. Nanobiotechnol., 2013, 11, 26.

32 N. A. Monteiro-Riviere, M. E. Samberg, S. J. Oldenburg and J. E. Riviere, Protein binding modulates the cellular uptake of silver nanoparticles into human cells: Implications for in vitro to in vivo extrapolations?, Toxicol. Lett., 2013, 220, 286-293.

33 A. E. Nel, et al., Understanding biophysicochemical interactions at the nano-bio interface, Nat. Mater., 2009, 8, 543-557. 
34 S. Laurent, C. Burtea, C. Thirifays, F. Rezaee and M. Mahmoudi, Significance of cell "observer" and protein source in nanobiosciences, J. Colloid Interface Sci., 2013, 392, 431-445.

35 X. He, W. G. Aker, P. P. Fu and H. M. Hwang, Toxicity of engineered metal oxide nanomaterials mediated by nanobio-eco-interactions: a review and perspective, Environ. Sci.: Nano, 2015, 2, 564-582.

$36 \mathrm{M}$. $\mathrm{Xu}$, et al., Formation of Nano-Bio-Complex as Nanomaterials Dispersed in a Biological Solution for Understanding Nanobiological Interactions, Sci. Rep., 2012, 2, 406.

37 A. R. Ribeiro, et al., Trojan-Like Internalization of Anatase Titanium Dioxide Nanoparticles by Human Osteoblast Cells, Sci. Rep., 2016, 6, 23615.

38 A. R. Ribeiro, et al., Bio-camouflage of anatase nanoparticles explored by in situ high-resolution electron microscopy, Nanoscale, 2017, 9, 10684-10693.

39 Z. Ji, X. Jin, S. George, T. Xia, H. Meng, X. Wang, E. Suarez, H. Zhang, E. M. V. Hoek, H. Godwin, A. E. Nel and J. I. Zink, Dispersion and Stability Optimization of $\mathrm{TiO}_{2}$ Nanoparticles in Cell Culture Media, Environ. Sci. Technol., 2010, 44(19), 7309-7314.

40 J. S. Taurozzi, V. A. Hackley and M. R. Wiesner, A standardised approach for the dispersion of titanium dioxide nanoparticles in biological media, Nanotoxicology, 2013, 7, 389-401.

41 J. S. Taurozzi, V. A. Hackley and M. R. Wiesner, Ultrasonic dispersion of nanoparticles for environmental, health and safety assessment - issues and recommendations, Nanotoxicology, 2011, 5, 711-729.

$42 \mathrm{~J}$. M. Robinson and M. J. Karnovsky, Ultrastructual localization of several phosphatases with cerium, $J$. Histochem. Cytochem., 1983, 31, 1197-1208.

$43 \mathrm{~W}$. Wu, et al., Dispersion Method for Safety Research on Manufactured Nanomaterials, Ind. Health, 2014, 52, 54-65.

44 M. Horie, et al., Protein Adsorption of Ultrafine Metal Oxide and Its Influence on Cytotoxicity toward Cultured Cells, Chem. Res. Toxicol., 2009, 22, 543-553.

45 R. Lamb and C. A. Ambler, Keratinocytes Propagated in Serum-Free, Feeder-Free Culture Conditions Fail to Form
Stratified Epidermis in a Reconstituted Skin Model, PLoS One, 2013, 8, e52494.

46 C. R. Kahl and A. R. Means, Regulation of Cell Cycle Progression by Calcium/Calmodulin-Dependent Pathways, Endocr. Rev., 2003, 24(6), 719-736.

47 P. Kocbek, K. Teskač, M. E. Kreft and J. Kristl, Toxicological Aspects of Long-Term Treatment of Keratinocytes with ZnO and $\mathrm{TiO}_{2}$ Nanoparticles, Small, 2010, 6, 1908-1917.

48 P. Ariano, P. Zamburlin, A. Gilardino, R. Mortera, B. Onida, M. Tomatis, M. Ghiazza, B. Fubini and D. Lovisolo, Interaction of Spherical Silica Nanoparticles with Neuronal Cells: Size-Dependent Toxicity and Perturbation of Calcium Homeostasis, Small, 2011, 7(6), 766-774.

49 J.-J. Yin, et al., Phototoxicity of nano titanium dioxides in HaCaT keratinocytes-Generation of reactive oxygen species and cell damage, Toxicol. Appl. Pharmacol., 2012, 263, 81-88.

50 D. J. Klionsky, E. L. Eskelinen and V. Deretic, Autophagosomes, phagosomes, autolysosomes, phagolysosomes, autophagolysosomes... Wait, I'm confused, Autophagy, 2014, 10(4), 549-551.

51 G. Van Niel, G. D'Angelo and G. Raposo, Shedding light on the cell biology of extracellular vesicles, Nat. Rev. Mol. Cell Biol., 2018, 19(4), 213-228.

52 R. E. Serda, A. Mack, A. L. van de Ven, S. Ferrati, K. Dunner Jr, B. Godin, C. Chiappini, M. Landry, L. Brousseau, X. Liu, A. J. Bean and M. Ferrari, Logic-embedded vectors for intracellular partitioning, endosomal escape, and exocytosis of nanoparticles, Small, 2010, 6(23), 2691-2700.

53 M. Mahmoudi, Debugging Nano-Bio Interfaces: Systematic Strategies to Accelerate Clinical Translation of Nanotechnologies, Trends Biotechnol., 2018, 36, 8.

54 M. Faria, et al., Minimum information reporting in bio-nano experimental literature, Nat. Nanotechnol., 2018, 13, 777785.

55 E. J. Guggenheim, et al., Refining in vitro models for nanomaterial exposure to cells and tissues, NanoImpact, 2018, 10, 121-142.

56 J. D. McBride, L. Rodriguez-Menocal and E. V. Badiavas, Extracellular Vesicles as Biomarkers and Therapeutics in Dermatology: A Focus on Exosomes, J. Invest. Dermatol., 2017, 137(8), 1622-1629. 